\title{
Immune profiling of plasma-derived extracellular vesicles identifies Parkinson disease
}

Elena Vacchi, MS, * Jacopo Burrello, MD, * Dario Di Silvestre, MS, Alessio Burrello, MS, Sara Bolis, MS, Pierluigi Mauri, PhD, Giuseppe Vassalli, MD, Carlo W. Cereda, MD, Cinthia Farina, PhD, Lucio Barile, PhD, Alain Kaelin-Lang, MD, PhD, and Giorgia Melli, MD, PhD

Neurol Neuroimmunol Neuroinflamm 2020;7:e866. doi:10.1212/NXI.0000000000000866

\section{Abstract}

\section{Objective}

To develop a diagnostic model based on plasma-derived extracellular vesicle (EV) subpopulations in Parkinson disease (PD) and atypical parkinsonism (AP), we applied an innovative flow cytometric multiplex bead-based platform.

\section{Methods}

Plasma-derived EVs were isolated from PD, matched healthy controls, multiple system atrophy (MSA), and AP with tauopathies (AP-Tau). The expression levels of $37 \mathrm{EV}$ surface markers were measured by flow cytometry and correlated with clinical scales. A diagnostic model based on EV surface markers expression was built via supervised machine learning algorithms and validated in an external cohort.

\section{Results}

Distinctive pools of EV surface markers related to inflammatory and immune cells stratified patients according to the clinical diagnosis. PD and MSA displayed a greater pool of overexpressed immune markers, suggesting a different immune dysregulation in PD and MSA vs AP-Tau. The receiver operating characteristic curve analysis of a compound EV marker showed optimal diagnostic performance for PD (area under the curve [AUC] 0.908; sensitivity 96.3\%, specificity 78.9\%) and MSA (AUC 0.974; sensitivity 100\%, specificity 94.7\%) and good accuracy for AP-Tau (AUC 0.718; sensitivity 77.8\%, specificity 89.5\%). A diagnostic model based on EV marker expression correctly classified $88.9 \%$ of patients with reliable diagnostic performance after internal and external validations.

\section{Conclusions}

Immune profiling of plasmatic EVs represents a crucial step toward the identification of biomarkers of disease for PD and AP.

\author{
Correspondence \\ Dr. Melli \\ giorgia.melli@eoc.ch
}




\section{Glossary}

AP = atypical parkinsonism; AP-Tau = atypical parkinsonism with tauopathies; AUC = area under the curve; BDI-II = Beck Depression Inventory II; CBD = corticobasal degeneration; EV = extracellular vesicle; HC = healthy control; H\&Y = Hoehn and Yahr scale; KEGG = Kyoto Encyclopedia of Genes and Genomes; LEDD = levodopa equivalent daily dose; MCSP = melanoma-associated chondroitin sulfate proteoglycan; MDS-UPDRS = Movement Disorder Society-Unified Parkinson's Disease Rating Scale; MFI = median fluorescence intensity; $\mathbf{M o C A}=$ Montreal Cognitive Assessment; $\mathbf{M S A}=$ multiple system atrophy; $\mathbf{n M F I}=$ normalized median fluorescence intensity; NTA = nanoparticle tracking analysis; PD = Parkinson disease; PPI = protein-protein interaction; PSP = progressive supranuclear palsy; RF = random forest; $\mathbf{R O C}=$ receiver operating characteristic; TSG101 = tumor susceptibility gene 101 .

To date, an effective causal treatment for Parkinson disease (PD) is missing, and the diagnosis still relies exclusively on motor symptoms that appear too late for a disease modifying intervention. ${ }^{1}$ Hence, there is urgent need for biomarkers that can stratify patients with PD for clinical trials. Furthermore, the differential diagnosis between $\mathrm{PD}$ and atypical parkinsonisms (APs) like multiple system atrophy (MSA) is challenging. ${ }^{2}$ According to the misfolded protein aggregates present in the brain, PD and MSA are collectively termed as alpha-synucleinopathies and are distinct from $\mathrm{AP}$ with tauopathies (AP-Tau).

Extracellular vesicles (EVs) are a heterogeneous population of secreted membrane particles involved into physiologic cell-tocell communication and transmission of biological signals. EVs are subdivided based on physical characteristics such as size, into small $(30-150 \mathrm{~nm})$ and large $(150-500 \mathrm{~nm})$ vesicles; members of the tetraspanin protein family (CD9, CD63, and CD81) are considered specific markers of EVs. ${ }^{3}$ CNS neurons release EVs ${ }^{4}$ able to cross the blood-brain barrier and reach the peripheral blood. ${ }^{5}$ EVs express surface antigens, which affect the cellular uptake and allow their tracking to the cell of origin. ${ }^{6}$

So far, most of the studies on EVs in neurodegenerative diseases focused on their possible role on transmission of pathologic misfolded proteins and fewer on their functions in cell-to-cell signaling. Indeed, immune system is involved in $\mathrm{PD}$, as demonstrated by neuroinflammatory changes in brain histopathology as well as by elevated immune markers in peripheral blood, suggesting that immune system may play a primary pathogenic role in $\mathrm{PD} .{ }^{7,8}$ Therefore, we hypothesized that circulating EVs carry important information on brain inflammatory immune response and that their characterization can be exploited for diagnostic purposes.

\section{Methods}

\section{Study design}

This was a cross-sectional, case-control study aiming (1) to characterize distinctive EV subpopulations in plasma of patients with PD, MSA, and AP-Tau healthy controls (HCs) by immunophenotyping 37 different membrane proteins using an innovative flow cytometry multiplex bead-based platform ${ }^{9,10}$; (2) to correlate the differential expression of EV surface antigens to clinical scales of gravity; and (3) to build diagnostic models based on distinctive EV surface proteins through supervised machine learning algorithms. Finally, because EVs are taken up by surrounding and distant cells, we performed a functional evaluation of their protein interactors with the purpose to highlight protein targets, biological pathways, and molecular functions potentially affected in PD, MSA, and AP-Tau.

\section{Subjects}

Twenty-seven patients with idiopathic PD, 8 with probable MSA, 9 with probable AP-Tau, and 19 age-matched HCs for the PD group were consecutively enrolled from July 2015 to January 2019. These subjects served as the training cohort for the diagnostic model.

Patients were recruited from the movement disorders outpatient clinic at Neurocenter of Southern Switzerland in Lugano; HCs were recruited among patients' partners. The inclusion criteria for PD were (1) a definite clinical diagnosis according to the UK Parkinson's Disease Society Brain Bank criteria for diagnosis ${ }^{1}$ and (2) no family history and no major cognitive impairment or major dysautonomic symptoms in the history. The inclusion criteria for AP were based on published diagnostic criteria for MSA, ${ }^{11}$ progressive supranuclear palsy (PSP), ${ }^{12}$ and corticobasal degeneration (CBD) ${ }^{13}$ Each subject underwent blood collection and clinical evaluation. Disease gravity was assessed by the Hoehn and Yahr scale (H\&Y) and Movement Disorder SocietyUnified Parkinson's Disease Rating Scale (MDS-UPDRS) during the off stage; cognitive profile by the Mini-Mental State Examination (MMSE) and Montreal Cognitive Assessment (MoCA); mood disorder by the Beck Depression Inventory II (BDI-II) scale; REM sleep behavior disorder (RBD) by the RBD screening questionnaire; and olfactory function by Burghart Messtechnik GmbH (olfactory test). Levodopa equivalent daily dose (LEDD) was calculated for patients with $\mathrm{PD}$ and $\mathrm{AP} .^{14}$

Exclusion criteria were significant comorbidities: diabetes, renal failure, thyroid pathology, vitamin B12 deficiency, HIV infection, syphilis, coagulopathy, fever, acute or chronic inflammatory diseases, and tumors. 
A separate cohort of 40 subjects (20 HC, 10 PD, 5 MSA, and 5 AP-Tau) served as the validation cohort for the diagnostic model (see below the paragraph "Diagnostic modeling and validation in an external cohort").

\section{Standard protocol approvals, registrations, and patient consents}

Subjects were consecutively included in the NSIPD001 study, according to the study protocol that was approved by the Cantonal Ethics Committee. All enrolled subjects gave written informed consent to the study in accordance with the Declaration of Helsinki.

\section{Blood collection and plasma preparation}

Ten milliliters of blood were collected into anticoagulant ethylenediamine tetraacetic acid (EDTA) tubes in the morning after 4-hour fasting, and the following protocol was performed to obtain plasma enriched in $\mathrm{EVs}^{15}$ : fresh whole blood was centrifuged for 15 minutes at $1,600 \mathrm{~g}$ at $10^{\circ} \mathrm{C}$ to eliminate cellular components. To further deplete platelets and cellular debris, the supernatant was centrifuged $15 \mathrm{mi}-$ nutes at $3,000 \mathrm{~g}$ at $4^{\circ} \mathrm{C}$; then, 2 consecutive centrifuges were performed at $10,000 \mathrm{~g}$ for 15 minutes and $20,000 \mathrm{~g}$ for $30 \mathrm{mi}-$ nutes at $4^{\circ} \mathrm{C}$, allowing the elimination of apoptotic bodies and larger EVs (figure 1A). The obtained plasma was aliquoted and stored at $-80^{\circ} \mathrm{C}$. The storage period varied among samples according to the consecutive enrollment of subjects in the study, between July 2015 and January 2019.

\section{Nanoparticle tracking analysis}

Nanoparticle concentration and diameter were measured by NanoSight LM10 (Malvern Instruments, Malvern, UK) equipped with a 405-nm laser and nanoparticle tracking analysis (NTA) 2.3 software. One microliter of plasma was diluted 1:1,000 in particle-free phosphate buffered saline. Three consequent videos of 60 seconds each were acquired. Minimum expected particle size, minimum track length, and blur setting were set to automatic, and the detection threshold was set to 4 to reveal all particles, as previously described. ${ }^{16}$ The particle concentration and the distribution graph of the particle size were determined per each sample by averaging the results from the analysis of 3 independent videos.

\section{MACSPlex exosome assay and flow cytometry analysis}

The screening approach (MACSPlex Human Exosome Kit; Miltenyi, Bergisch Gladbach, Germany) was previously described. ${ }^{9,10}$ Briefly, it is based on 4.8 - $\mu \mathrm{m}$ diameter polystyrene beads, labeled with different amounts of 2 dyes (phycoerythrin and fluorescein isothiocyanate), to generate 39 different bead subsets discriminable by flow cytometry analysis. Each bead subset is conjugated with a different capture antibody that recognizes EVs carrying the respective antigen (37 EV surface epitopes plus 2 isotype controls). The list of 37 antigens is reported in table e-1 (links.lww.com/NXI/A293). After beads + sample overnight incubation, EVs bound to beads are detected by allophycocyanin-conjugated anti-CD9, anti-
CD63, and anti-CD81 antibodies (figure 1A). Plasma samples $(60 \mu \mathrm{L})$ diluted 1:2 in buffer solution were analyzed with the MACSQuant Analyzer-10 flow cytometer (Miltenyi). Triggers for the side scatter and the forward scatter were selected to confine the measurement on the multiplex beads. A blank control composed only by MACSPlex Buffer and incubated with beads and detection antibodies was used to measure the background signal. Each EV marker's median fluorescence intensity (MFI) was normalized to the mean MFI for specific EV markers (CD9, CD63, and CD81) obtaining normalized MFI (nMFI). All analyses were based on nMFI values. Samples were analyzed blindly to the clinical diagnosis.

To test the reliability/specificity of MACSPlex Human Exosome Kit for EVs, we compared the procedure described above with and without $\mathrm{EV}$ enrichment by ultracentrifugation, and we found no differences between procedures (figure e-1, links.lww. com/NXI/A293). Therefore, plasma samples were directly processed without $\mathrm{EV}$ enrichment by ultracentrifugation.

Technical consistency and reproducibility of the assay were confirmed by analyzing repeatedly the same sample and by assessing plasma from the same subject at different time points (figure e-2, links.lww.com/NXI/A293).

\section{Western blot analysis}

Western blot analysis was performed on $100 \mu \mathrm{L}$ of plasma samples incubated overnight with $5 \mu \mathrm{L}$ of MACSPlex detection beads at $10^{\circ} \mathrm{C}$ at $800 \mathrm{rpm}$. The next day, the unbounded fraction was discarded, and samples were lysed with radioimmunoprecipitation assay buffer. Total proteins were separated on a gradient sodium dodecyl sulphatepolyacrylamide gel electrophoresis $4 \%-12 \%$ gel and transferred onto polyvinylidene difluoride membrane. The blot was incubated with the following primary antibodies: anti-Alix (rabbit polyclonal; Abcam, Cambridge UK, 1:1,000); antitumor susceptibility gene 101 (TSG101) (rabbit polyclonal; Abcam, 1:1,000); anti-CD81 (mouse monoclonal, Thermo Fisher Scientific, Waltham, MA, 1:300); anti-apolipoprotein A1 (APOA1) (rabbit polyclonal; Abcam, 1:300); and antiGRP94 (rabbit polyclonal; Abcam, 1:500).

\section{Network analysis of EV surface markers' protein interactors}

Protein interactors of differentially expressed EV surface markers were retrieved by Cytoscape PESCA plugin, ${ }^{17}$ and a global Homo sapiens protein-protein interaction (PPI) network of 1,588 nodes and 36,984 edges was reconstructed. For each quantitative comparison (PD vs HC, MSA vs HC, AP-Tau vs $\mathrm{HC}$ ), a specific PPI subnetwork was built considering the first neighbors of each EV surface protein. Each subnetwork was analyzed at a topological level by Cytoscape Centiscape plu$\operatorname{gin}^{18}$; to select putative hubs and bottlenecks, we took into account the network size, and only nodes with all Betweenness, Bridging, and Centroid values above the average calculated on the corresponding whole network were retained as previously reported. ${ }^{19,20}$ At the same time, nodes belonging to each 
A
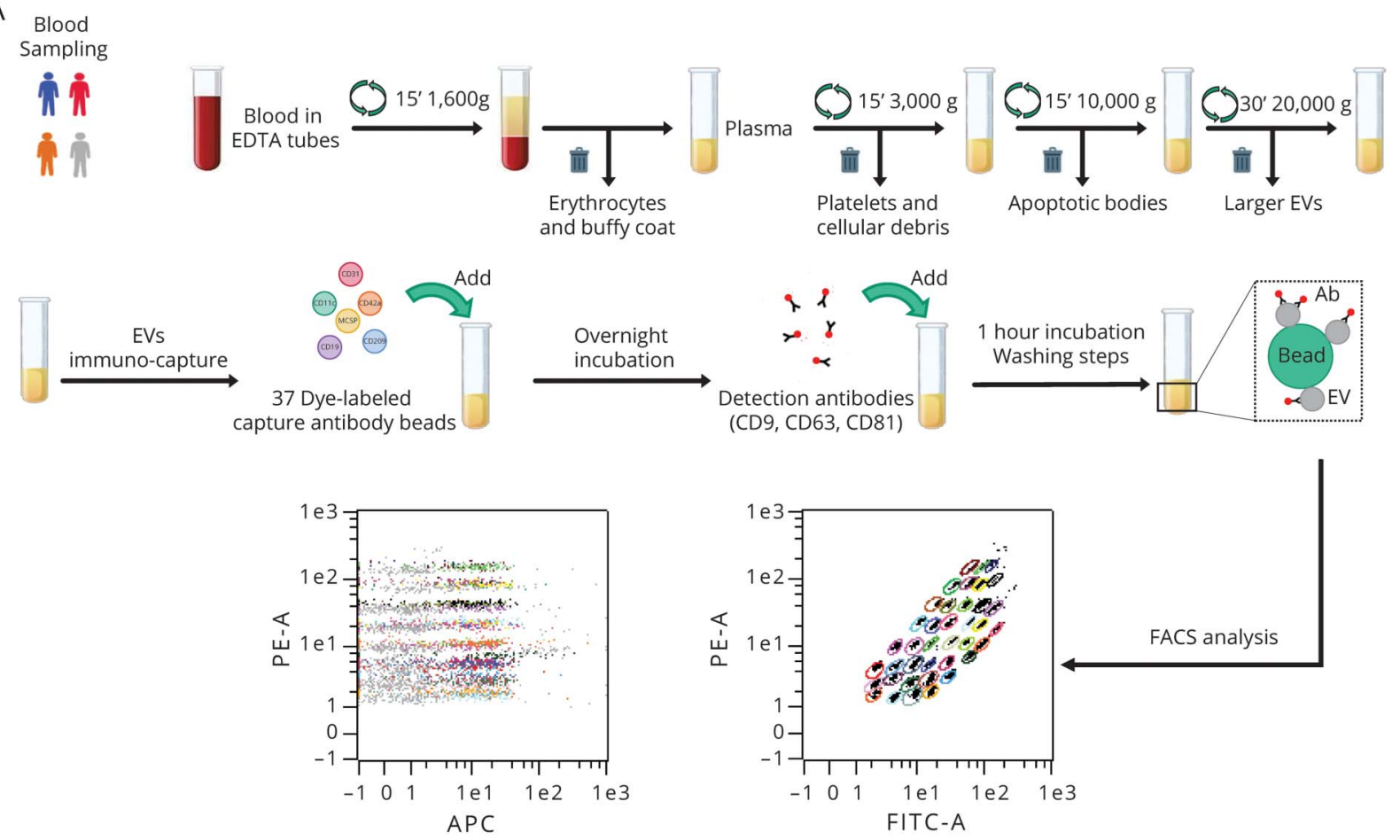

B

C
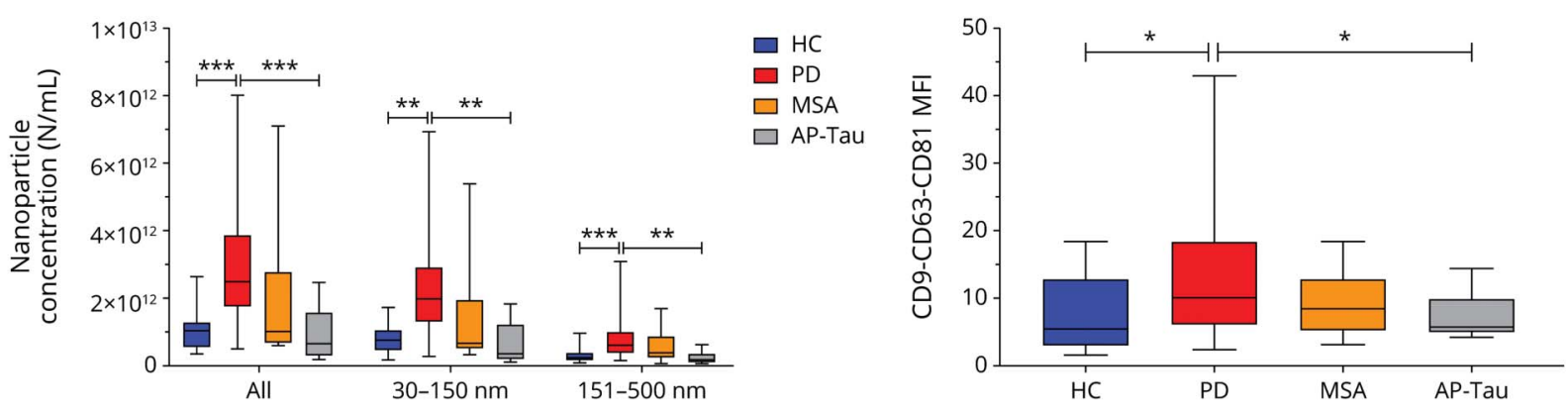

D

E
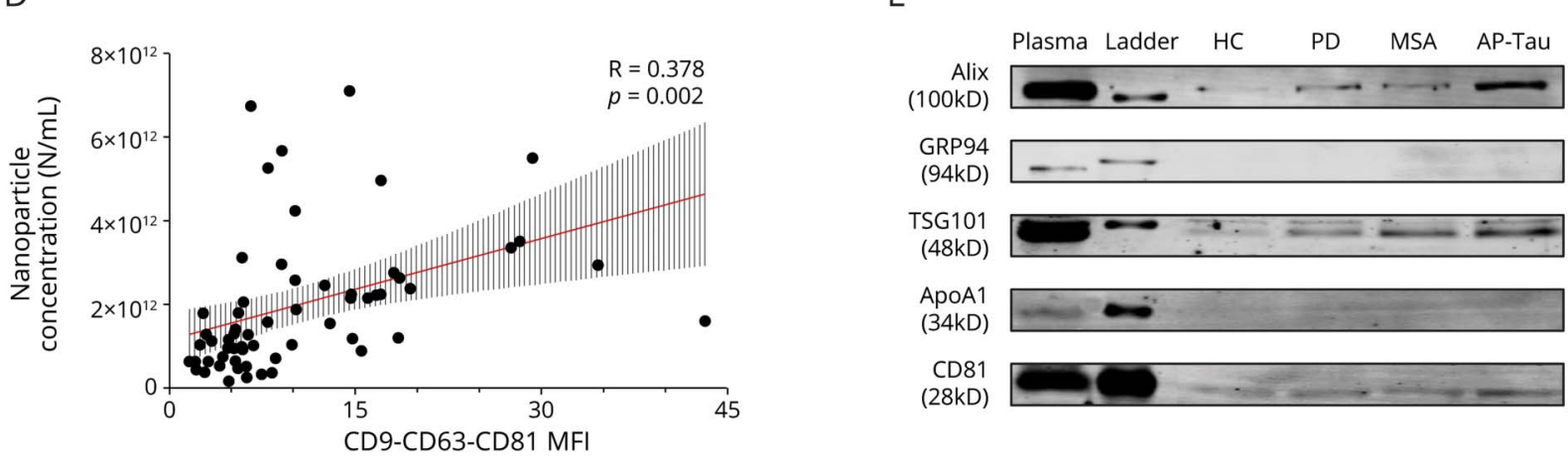

(A) Protocol for EV enrichment and MACSPlex exosome assay. Blood collected into anticoagulant EDTA tubes underwent serial centrifugation to eliminate cellular components and larger EVs. Plasma samples were incubated overnight with dye-labeled capture beads coated with antibodies against 37 different EV surface antigens. Detection antibodies against CD9, CD63, and CD81 were then added and incubated for 1 hour. After washing steps, samples were analyzed by flow cytometry. (B) Nanoparticle concentration ( $\mathrm{N} / \mathrm{mL}$ plasma) by nanoparticle tracking analysis (NTA), stratified for diameter (smaller nanoparticles, 30-150 nm; larger nanoparticles 151-500 nm). (C) Mean median fluorescence intensity (MFI) for CD9, CD63, and CD81 at flow cytometry analysis. (D) Correlation between mean MFI of $\mathrm{CD}^{-} \mathrm{CD}^{-} 3^{-} \mathrm{CD} 81$ and $\mathrm{N} / \mathrm{mL}$ by NTA: the regression line is reported in red, with $95 \% \mathrm{Cl}$. (E) Western blot of samples from $\mathrm{HC}$, PD, MSA, and AP-Tau subjects after immunocapturing compared with whole plasma (dilution 1:100), showing the presence of specific EV markers (CD81, Alix, tumor susceptibility gene 101) and the absence of plasma contaminants (apolipoprotein A1, GRP94). Data are expressed as median and interquartile range; $p$ values $<0.05$ were considered significant $(* p<0.05, * \star p<0.01, * \star \star p<0.001)$. AP-Tau $=$ atypical parkinsonism with tauopathies; $\mathrm{EV}=$ extracellular vesicle; $\mathrm{HC}=$ healthy control; MSA = multiple system atrophy; PD = Parkinson disease. 
subnetwork were evaluated at a functional level by DAVID ${ }^{21}$ and the most enriched Kyoto Encyclopedia of Genes and Genomes (KEGG) pathway databases. Molecular functions were extracted; specifically, $H$ sapiens set as background, count $>5$ and $p<0.001$, corrected by the Bonferroni test.

\section{Statistical analysis}

Statistical analyses were performed with IBM SPSS Statistics 22.0, PYTHON 2.7, and GraphPad PRISM 7.0a. Variable distribution was assessed by the Kolmogorov-Smirnov test. Normally distributed variables (age) were expressed as mean \pm SD and analyzed by the 1-way analysis of variance test with the post hoc Bonferroni test for multiple comparisons. Nonnormally distributed variables (disease duration, H\&Y, MDSUPDRS, BDI-II, MMSE, MoCA, olfactory test, RBD, LEDD, NTA, and MACSPlex analysis) were expressed as medians and interquartile range and analyzed using the Kruskal-Wallis test. Categorical variables (sex) were expressed as absolute number and percentage (\%) and analyzed by $\chi^{2}$ or Fisher exact tests. Univariate logistic regression analysis was performed to assess the ORs. Receiver operating characteristic (ROC) curve analysis was used to evaluate the area under the curve (AUC) and to compare diagnostic performances of selected variables. The Youden index $(\mathrm{J}=$ Sensitivity + Specificity -1$)$ was calculated to determine the cutoff with the greater accuracy. Correlations were evaluated by the Pearson $\mathrm{R}$ test and regression curve analysis; correlations were considered strong for $\mathrm{R}$ between $|1.0|$ and $|0.5|$, moderate between $|0.5|$ and $|0.3|$, and weak between $\mid 0.3$ and $|0.1|$. A $p$ value less than 0.05 was considered significant.

\section{Diagnostic modeling and validation}

Machine learning supervised algorithms are exploited in clinical practice to formulate predictions of selected outcomes based on a given set of labeled paired input-output training sample data. ${ }^{22,23}$ The linear discriminant analysis was used to build the 3D canonical plot (figure 2B); canonical components 1, 2, and 3 were calculated from weighted linear combinations of variables to maximize separation between the 4 groups (HC, PD, MSA, and AP-Tau); in the plot, each patient is represented by a point, the center of the spheres indicates the mean of (canonical 1; canonical 2; canonical 3) for each diagnosis, and spheres include patients with a linear combination coefficient that falls within the mean \pm SD (canonical $1 \pm S D$; canonical 2 $\pm \mathrm{SD}$; canonical $3 \pm \mathrm{SD}$ ). A diagnostic model was built through a random forest $(\mathrm{RF})$ classification algorithm on the training cohort $(\mathrm{n}=63)$; the algorithm created 20 different classification trees with a maximum number of 8 splits for each tree. The diagnosis derives from the outcome of each classification tree of the RF: for example, if at least 11 of 20 trees of the RF predict $\mathrm{PD}$, the patient will be classified as PD. The model was validated by a leave-one-out algorithm (internal validation) and in a different cohort $(n=40)$ (external validation). The leave-oneout validation was used to exclude overfitting bias and to evaluate generalizability of the model; briefly, the algorithm is trained on $n-1$ patients (where " $n$ " is the total number of patients), and the remaining patient is used to test the model.
The test patient is then changed and accordingly the training subgroup. The process is repeated a total of $\mathrm{n}$ times, with the test patient rotating at each round and the remaining subgroup used for model training. The external validation was performed with the same RF model trained on the training cohort.

\section{Data availability}

The raw data that support the findings of this article are available on request to the corresponding author.

\section{Results}

\section{Demographic and clinical characteristics of study groups}

Demographic data and clinical assessments for each group are summarized in table 1 . The MSA group included 4 MSA-C and 4 MSA-P; the AP-Tau group included 6 patients with probable PSP and 3 with possible CBD (table e-2, links.lww.com/NXI/ A293). Subjects with AP-Tau were significantly older than HC. Sex ratio and disease duration did not differ across groups. It is known that $\mathrm{AP}$ is characterized by a more aggressive disease course than PD; indeed, MSA and AP-Tau had a more severe disease gravity measured by the H\&Y and by the MDS-UPDRS; in addition, they displayed a higher cognitive impairment measured by the MMSE and MoCA. Finally, subjects with AP-Tau resulted more depressed than $\mathrm{PD}$ as measured by the BDI-II. LEDD was not different between groups of patients.

\section{The PD group shows an increased number of EVs}

NTA showed that the PD group had the highest number of nanoparticles $/ \mathrm{mL}$ compared with $\mathrm{HC}$ and AP-Tau ( $p=$ 0.001 ) not with MSA, whereas no differences in diameter were found between groups (figure $1 \mathrm{~B}$, table e-3, links.lww. com/NXI/A293). Because NTA is not specific for EVs, we used the MFI of CD9/CD63/CD81 (specific markers of $\mathrm{EVs}$ ) by flow cytometry analysis as a measure of $\mathrm{EV}$ concentration. Mean MFI of CD9/CD63/CD81 was significantly higher in PD compared with HC $(p=0.023)$ and AP-Tau $(p=$ 0.037 ) not with MSA (figure 1C). Importantly, mean MFI for CD9/CD63/CD81 correlated with nanoparticle concentration obtained with NTA analysis (figure 1D).

EVs were furtherly characterized according to current standard guidelines. ${ }^{3}$ After EV immunocapture by MACSPlex kit capture beads, we performed a Western blot analysis showing the presence of EV-specific luminal markers (TSG101, Alix), EV-specific tetraspanin (CD81), and the absence of contaminants (APOA1 and GPR94) (figure 1E). These results confirm the presence of EVs and the absence of relevant contamination in samples analyzed by flow cytometry.

\section{EV surface antigen expression differs between groups}

Among the 37 EV surface markers, 17 resulted differentially expressed between groups. Sixteen markers differed between PD and HC: T-cell (CD4), B-cell (CD19), leukocyte (CD45), 


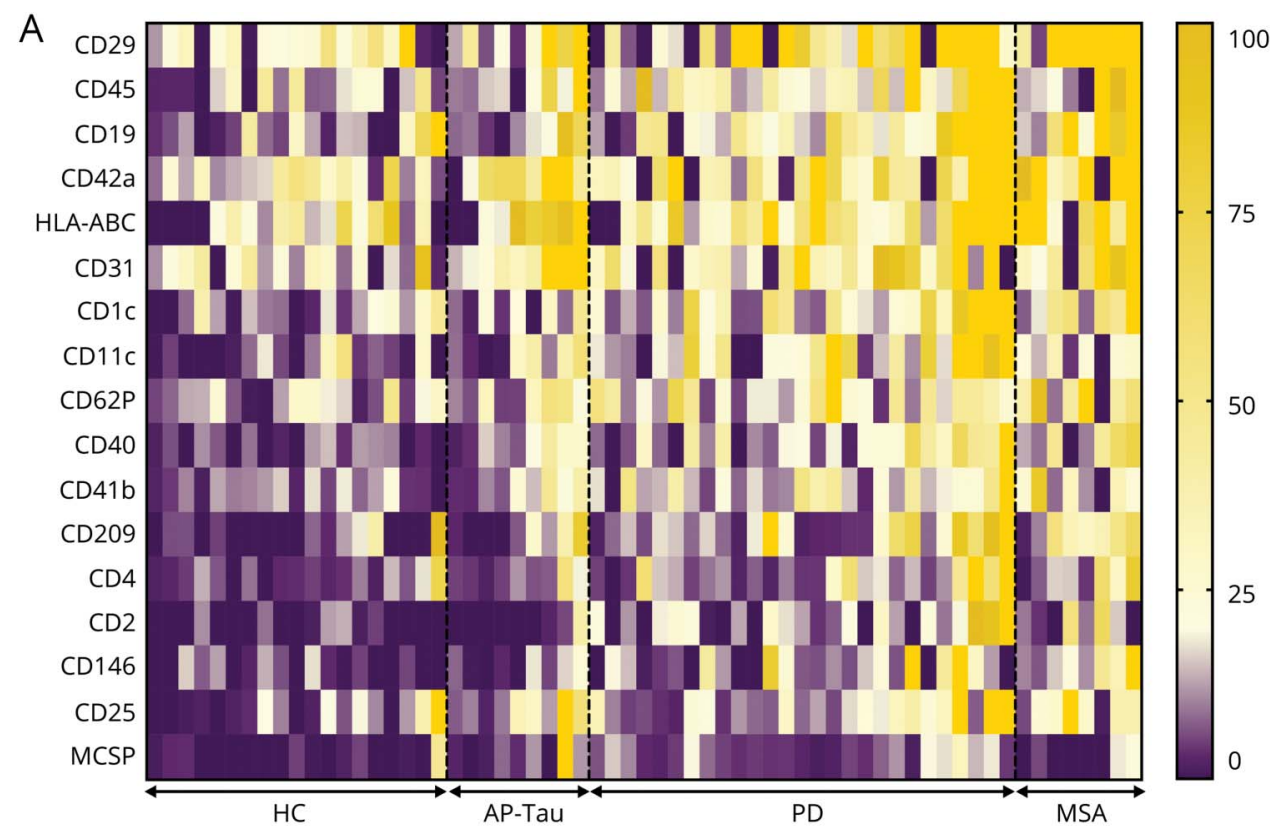

B

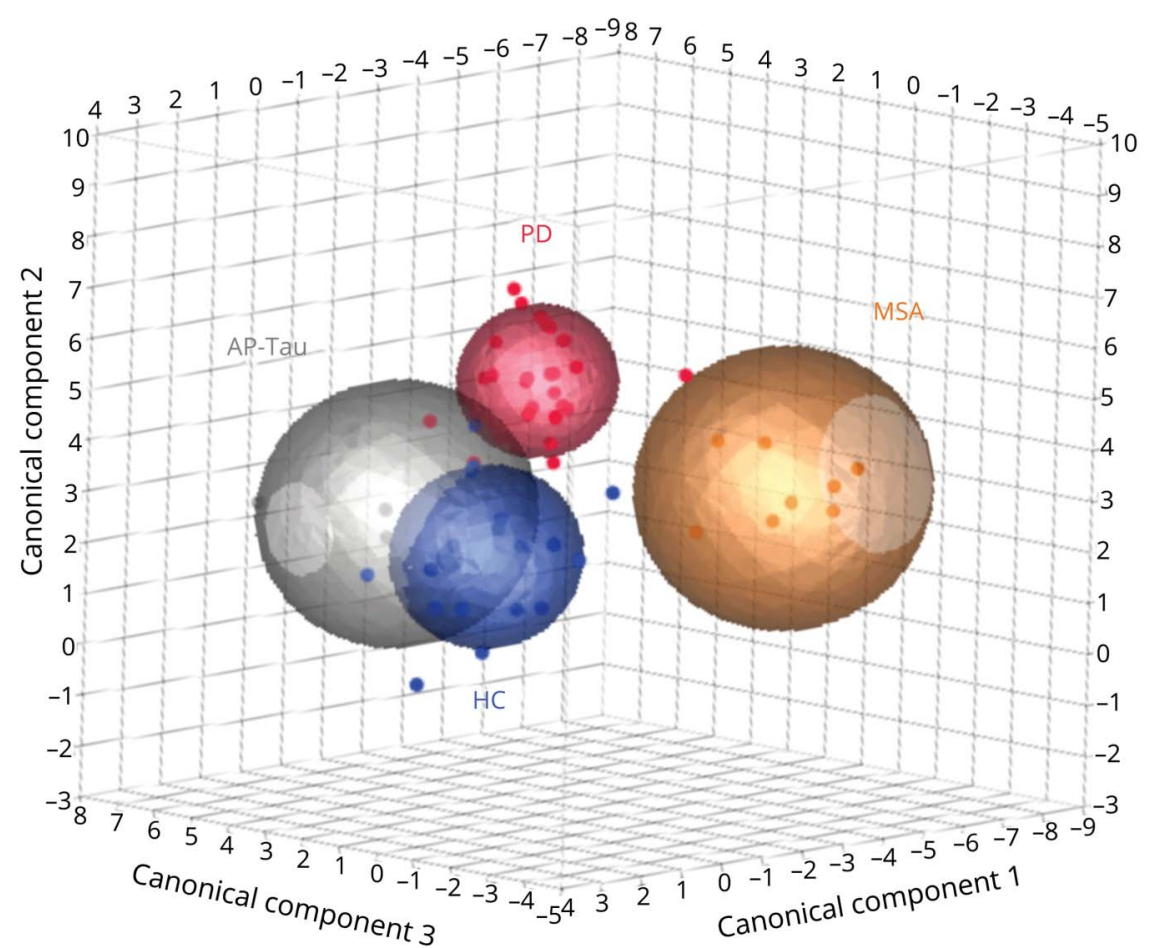

Patients' stratification for diagnosis and EV surface marker expression (expressed as normalized median fluorescence intensity [nMFI]). (A) Heatmap representation of the $17 \mathrm{EV}$ surface markers differentially expressed between patients with PD, MSA, and AP-Tau and HCs (purple = low nMFI, yellow = high nMFI). (B) Canonical plot showing patients according to the diagnosis: $P D$, red vs MSA, orange vs AP-Tau, gray vs HC, blue; the model was built considering the $37 \mathrm{EV}$ surface markers analyzed by flow cytometry. The axes of the plot (canonical 1, canonical 2, and canonical 3) were calculated from weighted linear combinations of variables to maximize separation between the 4 groups. Each subject is represented by a point, and spheres include patients with a linear combination coefficient that falls within the mean $\pm \mathrm{SD}$ (canonical $1 \pm \mathrm{SD}$; canonical $2 \pm S D$; canonical \pm SD). AP-Tau = atypical parkinsonism with tauopathies; $\mathrm{HC}=$ healthy control; MSA = multiple system atrophy; PD = Parkinson disease.

and antigen-presenting cell $(\mathrm{CD} 1 \mathrm{c})$ related markers, 8 involved in cell adhesion (CD2, CD11c, CD31, CD41, CD42a, CD62, $\mathrm{CD} 146$, and melanoma-associated chondroitin sulfate proteoglycan $[\mathrm{MCSP}]), 3$ in immune cell activation (CD25, CD40, and $\mathrm{CD} 209$ ), and the molecules of major histocompatibility complex class I human leukocyte antigen-ABC (HLA-ABC).

Twelve markers were different between MSA and HC: T cell related (CD4), B cell related (CD19), involved in cell adhesion (CD29, CD2, CD11c, CD31, CD42a, CD62, CD146, and
CD209), immune cell activation (CD40), and HLA-ABC. The AP-Tau and HC groups differ for only 4 markers: CD25, CD31, $\mathrm{CD} 40$, and CD42a. No significant difference was found between the PD and MSA groups, whereas CD2 resulted different between PD and AP-Tau. CD2 and CD19 discriminated between patients with MSA and AP-Tau. The nMFI of each EV marker in all groups is reported in table e-4 (links.lww.com/NXI/A293).

The heat map (figure 2A) built on the differentially expressed EV markers highlighted $\mathrm{HC}$ as a homogenous group with 


\begin{tabular}{|c|c|c|c|c|c|c|c|c|c|c|c|}
\hline \multirow[b]{2}{*}{ Variable } & \multirow[b]{2}{*}{$H C[n=19]$} & \multirow[b]{2}{*}{$P D[n=27]$} & \multicolumn{2}{|l|}{ AP } & \multirow[b]{2}{*}{$\begin{array}{l}\text { Overall } p \\
\text { value }\end{array}$} & \multicolumn{6}{|c|}{ Pairwise comparisons } \\
\hline & & & MSA $[n=8]$ & AP-Tau [n = 9] & & HC vs PD & $\begin{array}{l}\text { HC vs } \\
\text { MSA }\end{array}$ & $\begin{array}{l}\text { HC vs AP- } \\
\text { Tau }\end{array}$ & $\begin{array}{l}\text { PD vs } \\
\text { MSA }\end{array}$ & $\begin{array}{l}\text { PD vs AP- } \\
\text { Tau }\end{array}$ & $\begin{array}{l}\text { MSA vs AP- } \\
\text { Tau }\end{array}$ \\
\hline Age (y) & $61 \pm 8.2$ & $66 \pm 11.8$ & $68 \pm 8.6$ & $74 \pm 5.2$ & 0.013 & 0.556 & 0.729 & 0.008 & 1.000 & 0.184 & 0.924 \\
\hline Sex (ref. male) & $10(52.6 \%)$ & $17(63.0 \%)$ & $2(25.0 \%)$ & $4(44.4 \%)$ & 0.279 & - & - & - & - & - & - \\
\hline Disease duration (y) & - & $4.0[2.0-8.0]$ & $5.5[1.3-7.8]$ & $4.0[2.5-5.5]$ & 0.863 & - & - & - & - & - & - \\
\hline H\&Y & - & $2.0[1.0-3.0]$ & $5.0[4.0-5.0]$ & $4.0[3.0-5.5]$ & $<0.001$ & - & - & - & $<0.001$ & 0.001 & 1.000 \\
\hline MDS-UPDRS & - & $23.0[13.0-34.5]$ & $42.5[38.0-43.0]$ & $40.5[28.8-81.5]$ & 0.043 & - & - & - & 0.509 & 0.277 & 1.000 \\
\hline BDI-II & - & $5.0[3.0-8.5]$ & $8.0[2.8-15.8]$ & $14.5[11.8-19.0]$ & 0.008 & - & - & - & 1.000 & 0.012 & 0.682 \\
\hline MMSE & - & $30.0[29.0-30.0]$ & $26.0[24.0-29.0]$ & $26.0[22.0-28.0]$ & $<0.001$ & - & - & - & 0.049 & 0.002 & 1.000 \\
\hline MoCA & - & $27.0[23.8-29.0]$ & $24.5[17.3-27.0]$ & $20.0[14.5-23.0]$ & 0.016 & - & - & - & 0.955 & 0.041 & 1.000 \\
\hline Olfactory test & - & $7.0[4.0-9.0]$ & $9.0[7.5-10.3]$ & $7.0[4.8-8.8]$ & 0.131 & - & - & - & - & - & - \\
\hline RBD & - & $3.0[1.8-5.0]$ & $3.0[1.0-5.8]$ & $3.0[0.5-4.5]$ & 0.875 & - & - & - & - & - & - \\
\hline LEDD & - & $\begin{array}{l}562.5 \\
{[202.5-737.5]}\end{array}$ & $\begin{array}{l}375.0 \\
{[108.0-375.0]}\end{array}$ & $\begin{array}{l}250.0 \\
{[100.0-452.0]}\end{array}$ & 0.448 & - & - & - & - & - & - \\
\hline $\begin{array}{l}\text { Abbreviations: } A P=\text { aty } \\
\text { MDS-UPDRS = Movem } \\
\text { RBD = REM sleep beha } \\
\text { Clinical characteristics }\end{array}$ & $\begin{array}{l}\text { parkinsonis } \\
\text { isorder Socie } \\
\text { disorder scre } \\
\text { tients with P }\end{array}$ & $\begin{array}{l}\text {; AP-Tau = atypical } \\
\text { y-Unified Parkinsol } \\
\text { ening questionnaire } \\
\text {, MSA, and AP-Tau }\end{array}$ & $\begin{array}{l}\text { kinsonism with tai } \\
\text { Disease Rating Scal } \\
\text { npared with HCs. }\end{array}$ & $\begin{array}{l}\text { athies; } \mathrm{BDI}-\mathrm{II}=\mathrm{Beck} \\
\mathrm{MSE}=\mathrm{Mini}-\text { Mental } \\
\text { lues }<0.05 \text { were co }\end{array}$ & $\begin{array}{l}\text { pression Inv } \\
\text { te Examinatic } \\
\text { lered signific }\end{array}$ & $\begin{array}{l}\text { II; } \mathrm{HC}=\text { heal } \\
\mathrm{CA}=\text { Montre } \\
\text { d shown in }\end{array}$ & $\begin{array}{l}\text { hy contr } \\
\text { l Cognit } \\
\text { old. }\end{array}$ & $\begin{array}{l}\text { = Hoehn an } \\
\text { essment; MS }\end{array}$ & $\begin{array}{l}\text { hr scale; } \\
\text { nultiple s) }\end{array}$ & $\begin{array}{l}=\text { levodopa } \\
\text { hatrophy; PL }\end{array}$ & $\begin{array}{l}\text { /alent daily dos } \\
\text { arkinson diseas }\end{array}$ \\
\hline
\end{tabular}


relatively low expression of EV markers, in analogy to the APTau group, whereas PD and MSA were characterized by higher levels of expression.

Furthermore, a linear discriminant analysis model based on differential expression of all EV markers allowed the separation of subjects according to their diagnosis, as shown in the canonical plot (figure 2B).

\section{Protein network hubs and functional pathway analysis of EV surface antigens}

The most relevant interactors of differentially expressed EV markers were selected by PPI network topological analysis in terms of hubs. Hubs refer to proteins with the greater number of connections within the cell or occupying crucial network positions, suggesting therefore a critical role on the control of information flow over the network. ${ }^{20,24}$ Analysis of hubs takes account of networks size and only nodes with values above the average normalization for the level of connections in the total network are selected. ${ }^{19,20}$ Hubs for PD vs HC and MSA vs HC comparisons were coincident (SP1, MSN, ITGB2, EZR, C1QBP, and CARL), whereas the network on AP-Tau vs HC showed a different set of proteins (FLNA, FN1, GP1BB, HSPA4, NFKB1, STAT3, VIM, VWF, and YWHAZ) (figure 3, A-C, table e-5, links.lww.com/NXI/A293). Similarities between PD and MSA were observed also in terms of pathways and molecular functions (figure 3D, table e-6). Most represented KEGG categories included immune system, signal transduction, endocrine system, and signaling molecules and interaction. Except for the endocrine system, they were more enriched in PD and MSA, suggesting potential stronger activation of immune response in these groups. Of note, FoxO signaling pathway was higher in AP-Tau.

\section{EV surface antigens correlate with cognitive} impairment and disease gravity in PD and MSA In PD there was a negative correlation between CD25 and MMSE and MoCa scores, a negative correlation between CD146 and MMSE score, whereas CD62P directly correlated with the BDI-II (figure 4, A-D). No significant correlations were found between EV antigen's expression and LEDD in the PD and AP groups (table e-7, links.lww.com/NXI/A293). In the MSA group, mean MFI for CD9, CD63, and CD81 inversely correlated with the MoCA, whereas nanoparticle concentration directly correlated with the disease duration and CD31 inversely correlated with the H\&Y (figure 4, E-G). No correlations were observed in the AP-Tau group (table e-7).

\section{Differential EV surface antigen expression and diagnostic outcome}

Univariate logistic regression analysis, allowing the assessment of associations between each EV marker and the diagnosis, confirmed $11 \mathrm{EV}$ surface antigens as potential discriminants for PD diagnosis, 6 for MSA, and 3 for AP-Tau (figure e-3, links.lww.com/NXI/A293). Among markers significantly associated with the different diagnoses, CD1c,
CD11c, CD19, CD41b, CD45, and CD146 were exclusive of the PD group; CD29 was exclusive for the MSA group. CD2 displayed the strongest association with the diagnosis of $\mathrm{PD}$ $(\mathrm{OR}=1.191)$ and MSA $(\mathrm{OR}=1.256)$, whereas CD40 with AP-Tau $(\mathrm{OR}=1.131)$.

\section{ROC curve analysis of EV surface antigens shows best performance for MSA and PD}

ROC curve analysis for all pathologic groups (PD, MSA, and AP-Tau) vs HC confirmed a reliable diagnostic performance of each single differentially expressed EV markers (figure 5, table e-8, links.lww.com/NXI/A293). The linear weighted combination of the 3 markers with the highest AUC showed better diagnostic performance respect to single markers in all groups. The combination of all EV surface markers in 1 single compound marker showed a further diagnostic improvement in the PD and MSA groups.

\section{Random forest model discriminates the different groups}

An RF diagnostic model was built using the 17 surface antigens differentially expressed in plasma-derived EVs. The forest was composed by 20 different classification trees (a representative tree is reported in figure $6 \mathrm{~A}$ ). The model discriminated patients of the 4 different groups (HC, PD, MSA, and AP-Tau) with high accuracy (88.9\%): all subjects with PD were correctly diagnosed, and $1 \mathrm{MSA}$ and $1 \mathrm{HC}$ were, respectively, misdiagnosed as $\mathrm{HC}$ and $\mathrm{PD}$, whereas among 9 patients with AP-Tau, 2 were predicted as $\mathrm{HC}$ and 3 as $\mathrm{PD}$ (figure 6B). Subsequently, pairwise comparisons were performed (figure 6, C-H). The RF model was validated by the leave-one-out algorithm, which confirmed the generalizability of the model and excluded overfitting bias (accuracy of internal validation $63.8 \%$, with a $72.2 \%-91.5 \%$ range for pairwise comparisons). Finally, we validated our model in an external cohort of 40 subjects: the overall accuracy was $77.5 \%$, resulting in the correct diagnosis of 31 of 40 subjects (figure 6, $\mathrm{I}$ and $\mathrm{J}$ ). The accuracy after external validation was consistent with the one resulting from the internal validation, supporting the reliability of the diagnostic model. Demographic data of the external cohort were similar to those of the training cohort and are shown in table e-9 (links.lww.com/NXI/A293).

\section{Discussion}

The major finding of this study consists in the setup of a diagnostic model for the stratification of patients with PD and AP, based on immunologic profiling of plasmatic EV subpopulations, obtained from minimally invasive peripheral blood sampling. We systematically evaluated the diagnostic performance of differentially expressed $\mathrm{EV}$ antigens, and a diagnostic model was built using supervised machine learning algorithms. The model showed an overall reliable accuracy, correctly predicting patient diagnosis, with the best performance for the diagnosis of PD (97.8\%) and MSA (100\%) vs HC. These results were supported by ROC curve analysis on the compound marker, originated from the linear combination of all the 
Figure 3 Extracellular vesicle (EV) surface proteins upregulated in PD, MSA, and AP-Tau and functional evaluation of their protein interactors

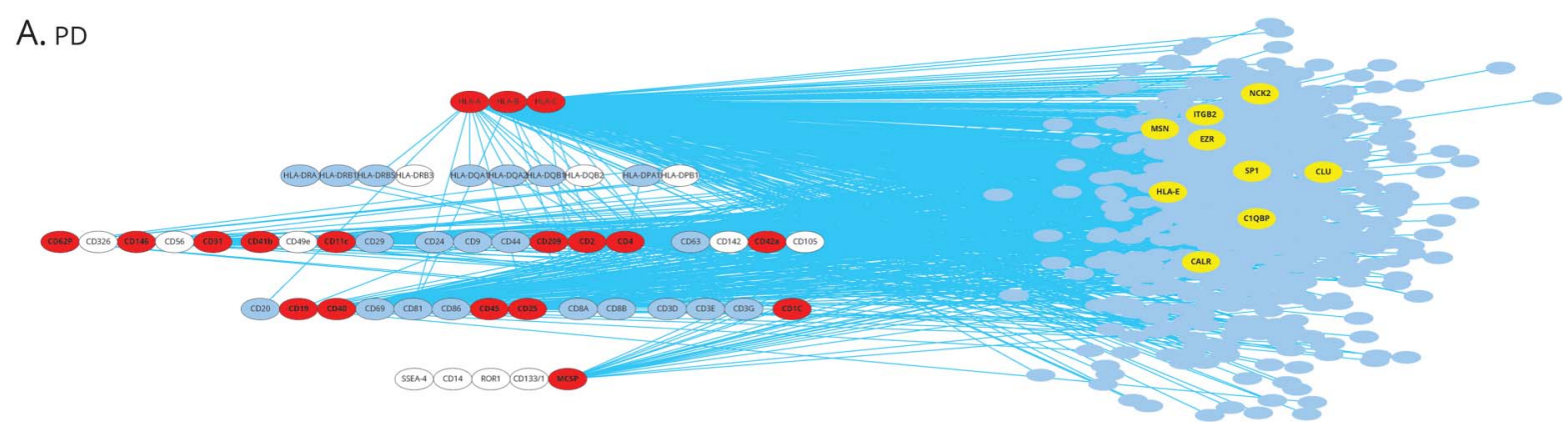

B. MSA

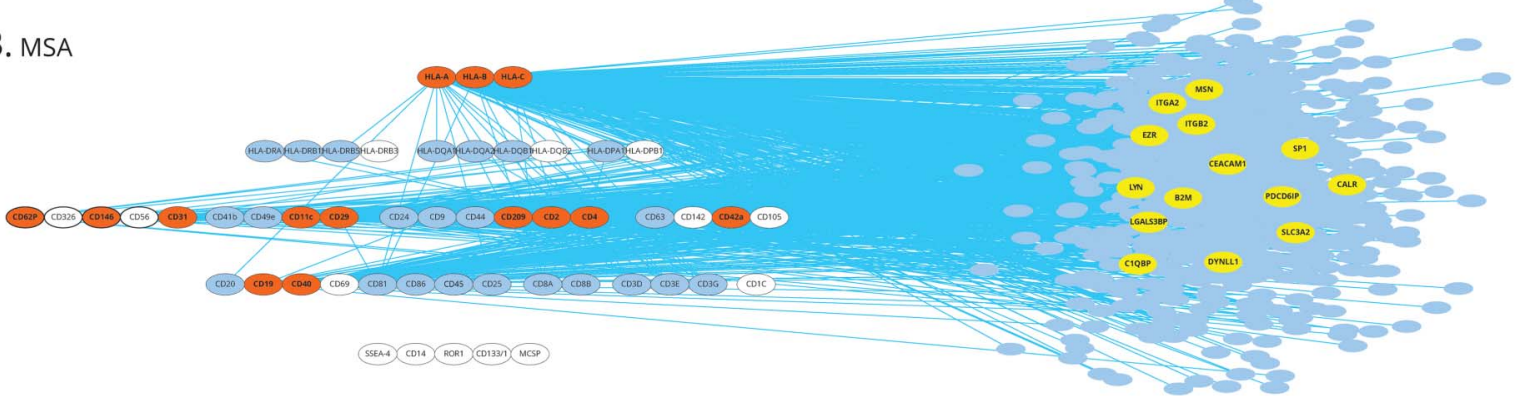

C. AP-Tau

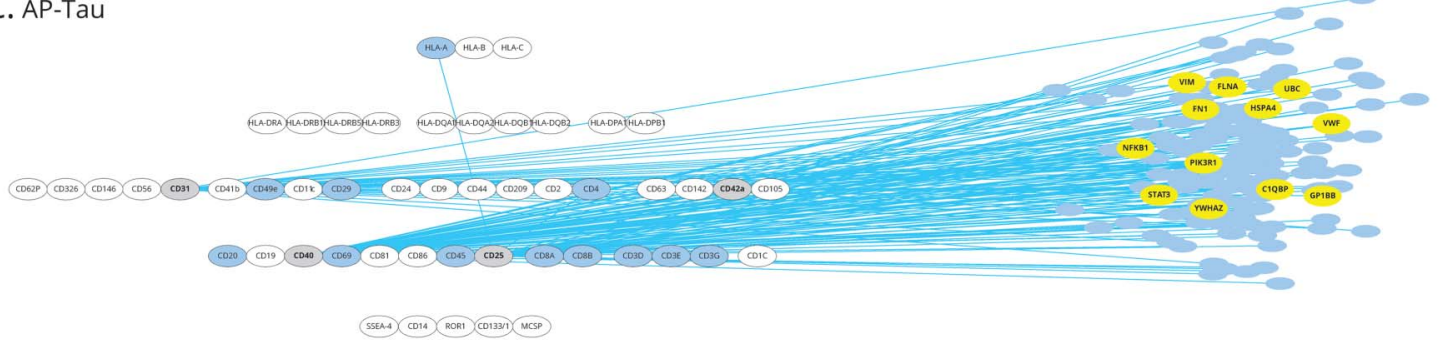

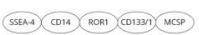

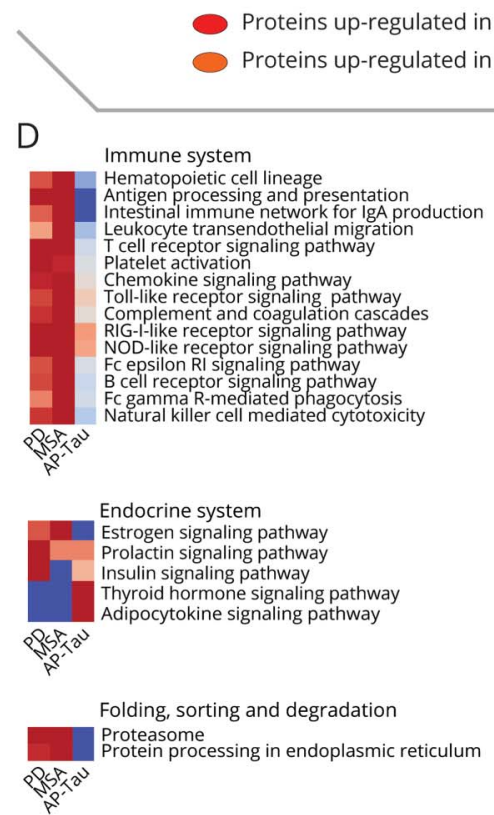

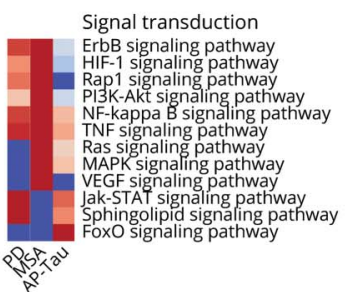

Excretory system

Endocrine and factor-regulated CA reabsorbtion Aldosterone-regualted sodium reabsorption $20250^{2}$

Other kegg categories

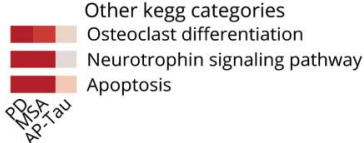

Signaling molecules and interaction cellular community, cell motility

Cell adhesion molecules (CAMs)

Focal adhesion

ECM-receptor interaction
Regulation of actin cytoskeleton Adherens junction

Transport and catabolism

Endocytosis

estasos

no. genes (\%)

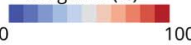

PPI network showing the first neighbors of each differentially expressed EV surface marker in (A) PD, (B) MSA, and (C) AP-Tau vs HC. (D) Kyoto Encyclopedia of Genes and Genomes pathways enriched by considering the first neighbors of each EV surface protein in PD, MSA, and AP-Tau vs HC; DAVID database background: Homo sapiens, gene count $>5$ and $p<0.001$. AP-Tau = atypical parkinsonism with tauopathies; $\mathrm{HC}=$ healthy control; MSA $=$ multiple system atrophy; PD = Parkinson disease; PPI = protein-protein interaction. 


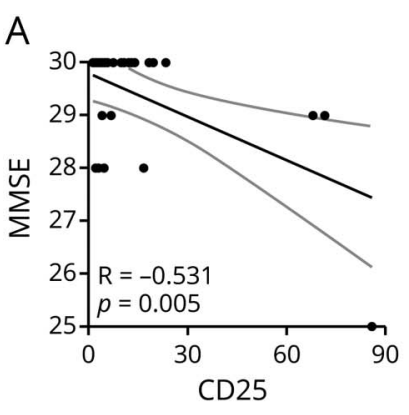

E

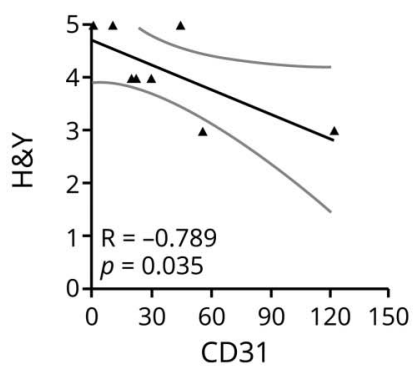

B

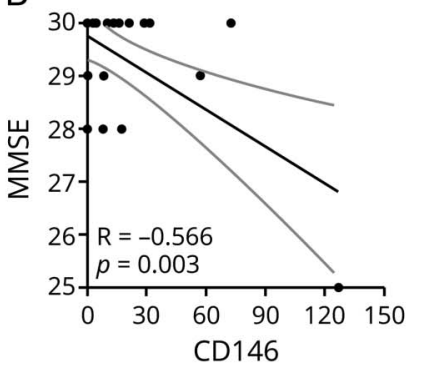

F

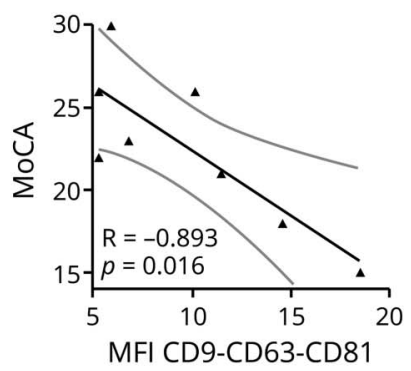

C

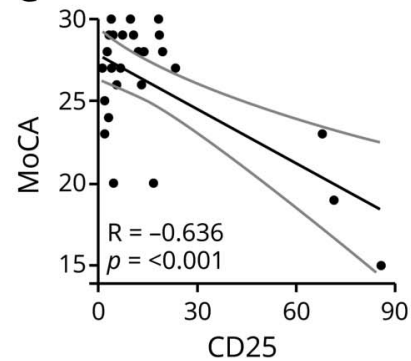

G

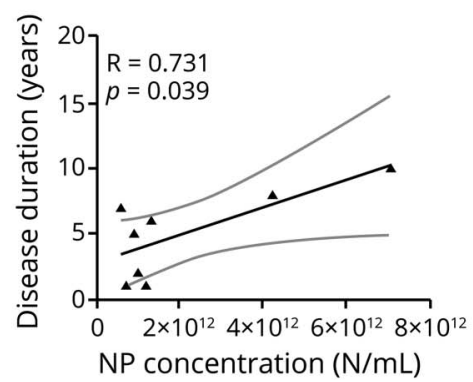

Correlations between EV surface markers normalized MFI, nanoparticle concentration (N/mL plasma), and clinical parameters in patients with Parkinson disease (circles; A-D) and multiple system atrophy (triangles; E-G). The regression line is reported together with its $95 \% \mathrm{CI}$ (dashed line). BDI-II = Beck Depression Inventory II; H\&Y = Hoehn and Yahr scale; MFI = median fluorescence intensity; MMSE = Mini-Mental State Examination; MoCA = Montreal Cognitive Assessment; RBD = REM sleep behavior disorder screening questionnaire.

differentially expressed EV markers, showing very high sensitivity and specificity for PD and MSA (AUC 0.908 and 0.974, respectively). Previous works have explored the utility of EVs as biomarkers for PD by quantifying brain-derived exosomes (AUC $0.75-0.82)^{25}$ or by measuring specific target proteins like alpha-synuclein $(\alpha \mathrm{Syn})$ or DJ-1 in plasma neuronal-derived exosomes (AUC $0.654,0.724$ ). ${ }^{26}$ The combination of multiple markers improved the diagnostic accuracy of neuronal-derived exosomes as shown by a recent work on quantification of both aSyn and clusterin differentiating PD from other proteinopathies and from MSA with high accuracy (AUC 0.98 and 0.94, respectively). ${ }^{27}$

This analysis of multiple immune surface markers of circulating EVs in $\mathrm{PD}$ and $\mathrm{AP}$ shows a high diagnostic performance, likely due to the advantage of simultaneously profiling several EV subpopulations. First of all, we demonstrated that plasma EV concentration was higher in patients with PD. Previous reports have shown that the total number and size of EVs were not augmented in serum of $\mathrm{PD},{ }^{28}$ whereas a more recent study demonstrated an increased number of plasmatic brain-derived EVs in PD. ${ }^{25}$ Methodological factors such as isolation/extraction and quantification of EVs explain these differences. However, at the molecular level, it is recognized that endosome/lysosome pathway is a common defective pathway in sporadic and genetic $\mathrm{PD},{ }^{29}$ and $\mathrm{EV}$ s are generated and secreted by the endosomal compartment called multivesicular bodies by fusion with plasma membrane. The process of EV secretion may be enhanced when there is an inhibition of fusion of multivesicular bodies with lysosomes, as expected in $\mathrm{PD},{ }^{30}$ so that an increased production of EVs in PD is likely.

It is difficult to track the origin of EVs because the majority of markers are shared by several cell types and virtually any cell can release EVs in blood. In blood normally, a large number of EVs arises from platelets, erythrocytes, however leucocytes, endothelial cells, monocytes, neutrophils and lymphocytes may release EVs. ${ }^{31}$ The flow cytometry analysis demonstrated that 16 and $12 \mathrm{EV}$ markers, related to immune cells, were upregulated respectively in PD and MSA, only 4 in AP-Tau compared with healthy condition. In particular, PD and MSA shared $11 \mathrm{EV}$ surface markers. Considering functions and roles of EV surface markers analyzed in this study, this result favors the hypothesis of a major, or at least different, immune dysregulation in PD and MSA vs AP-Tau. Despite sharing several overlapping clinical features, synucleinopathies and tauopathies are distinguished by distinctive neuropathologic hallmarks: deposits of aggregated aSyn (Lewy bodies) in neurons and in glial cells in the former group and neurofibrillary tangles of Tau in the latter as shown by immunohistological studies. ${ }^{32}$ Although inflammatory features have been described in patients with both synucleinopathies and tauopathies, by PET studies, ${ }^{33-36}$ the pathways activated are probably different. Animal studies have shown that the neurotoxic effects of beta-amyloid aggregates in a model of tauopathy (Alzheimer disease) are mediated via Toll-like receptor 4-dependent glial cell activation, while $\alpha$ Syn aggregates in a model of PD, activated Toll-like receptor 2 independently 


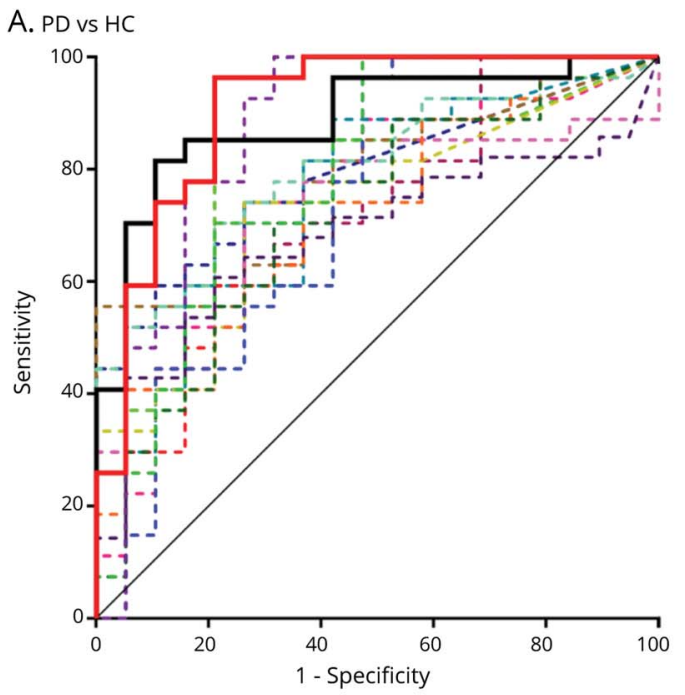

$$
\begin{aligned}
& \begin{array}{llll}
-\cdots & \mathrm{CD} 1 \mathrm{C} & -\cdots & \mathrm{CD} 45
\end{array} \\
& \text {-.. } \mathrm{CD} 2 \text {-.. } \\
& \text {-.. CD4 H. CD146 } \\
& \text {-.. CD11C - } \\
& \text {-.. CD19 -.. MCSP } \\
& \text {-.. CD25 -.. HLA-ABC } \\
& \text {-.. CD31 - CD2 + CD40 + MCSP }
\end{aligned}
$$

\begin{tabular}{|c|c|c|}
\hline & CD2+CD41b+MCSP & Compound marker \\
\hline Asymptotic significance & 0.000 & 0.000 \\
\hline AUC (95\% CI) & $0.889(0.792-0.986)$ & $0.908(0.815-1.000)$ \\
\hline Sensitivity (\%) & 81.5 & 96.3 \\
\hline Specificity (\%) & 89.5 & 78.9 \\
\hline
\end{tabular}

B. MSA vs HC

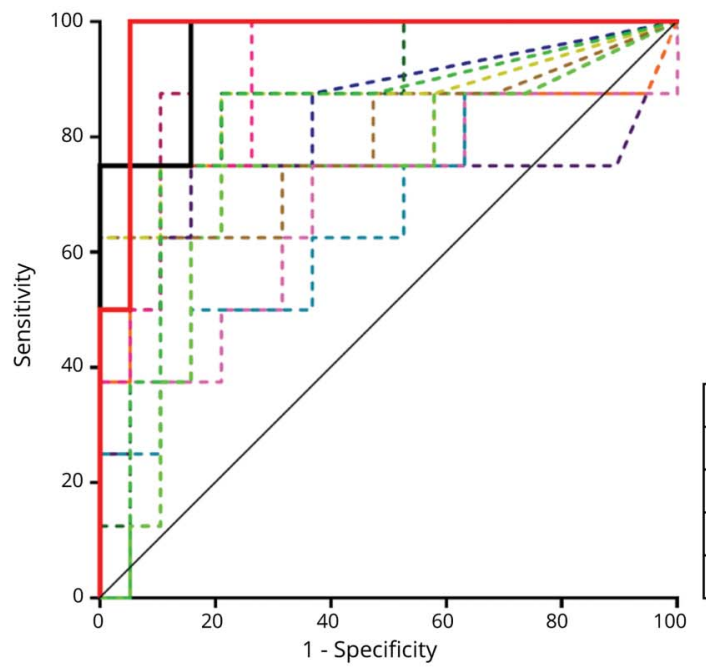

$$
\begin{aligned}
& \text {-.. CD2 - C. CD42a } \\
& \text {-.. } \mathrm{CD} 4 \quad \ldots \cdot \mathrm{CD} 62 \mathrm{P} \\
& \text {-.. CD11C - CD CD146 } \\
& \text {-.. CD19 --. CD209 } \\
& \text {-.. CD19 - C. HLA-ABC } \\
& \text {-.. CD25 - CD2+CD62P + CD146 } \\
& \text {-.. CD31 Combination of all } \\
& \text {-.. CD40 - EV-surface markers }
\end{aligned}
$$

C. AP-Tau vs HC

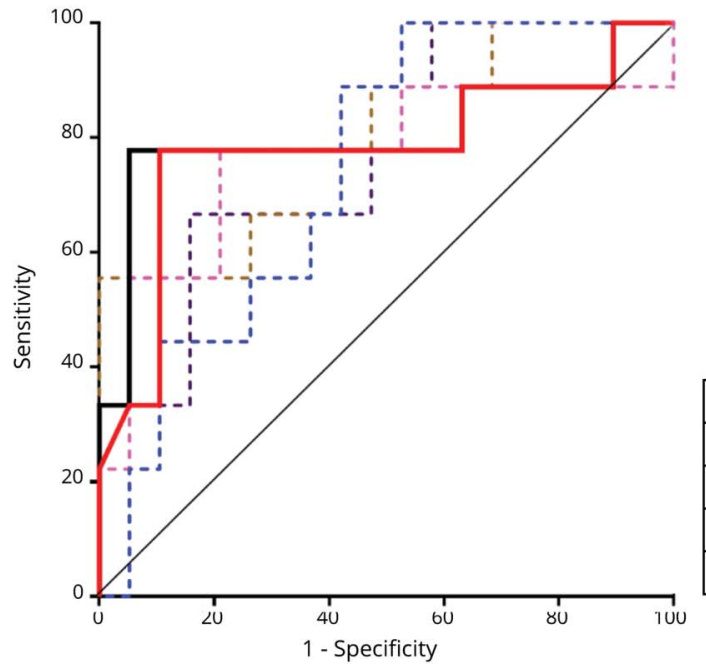

\begin{tabular}{|c|c|c|}
\hline & CD2+CD62P+CD146 & Compound marker \\
\hline Asymptotic significance & 0.000 & 0.000 \\
\hline AUC (95\% CI) & $0.961(0.893-1.000)$ & $0.974(0.917-1.000)$ \\
\hline Sensitivity (\%) & 100 & 100 \\
\hline Specificity (\%) & 84.2 & 94.7 \\
\hline
\end{tabular}

-.. CD31

-.. CD25

--. CD40

-.. CD42a

- $\mathrm{CD} 31+\mathrm{CD} 40+\mathrm{CD} 42 \mathrm{a}$

— Combination of all

- EV-surface markers

\begin{tabular}{|c|c|c|}
\hline & CD31+CD40+CD42a & Compound marker \\
\hline Asymptotic significance & 0.010 & 0.018 \\
\hline AUC (95\% CI) & $0.807(0.592-1.000)$ & $0.718(0.566-0.995)$ \\
\hline Sensitivity (\%) & 77.8 & 77.8 \\
\hline Specificity (\%) & 94.7 & 89.5 \\
\hline
\end{tabular}

ROC curves identifying the best cutoff for each EV surface marker, discriminating pathologic groups from HC. The referral line is reported in gray. (A) PD vs HC; (B) MSA vs HC; (C) AP-Tau vs HC. In each plot, ROC curves for the combination of the 3 EV surface markers with the highest AUCs and for a compound EV marker (linear weighted combination of all EV surface markers differentially expressed for each comparison) are shown (black and red lines, respectively). The tables provide asymptotic significance AUC with $95 \% \mathrm{Cl}$, sensitivity, and specificity on the compound EV markers. $p$ Values $<0.05$ were considered significant. AP-Tau = atypical parkinsonism with tauopathies; $\mathrm{AUC}=$ area under the curve; $\mathrm{HC}=$ healthy control; $\mathrm{MSA}=$ multiple system atrophy; PD = Parkinson disease. 
Figure 6 Random forest (RF) modeling to predict diagnosis and its validation in an external cohort of subjects

A

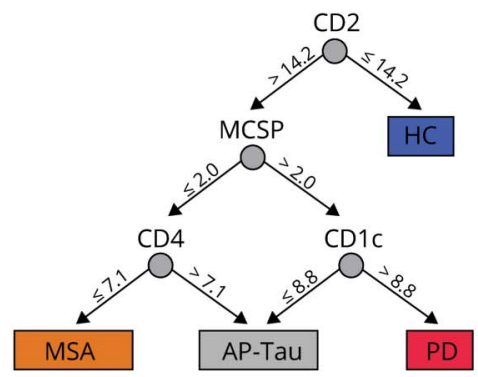

C

\begin{tabular}{|c|c|c|c|}
\hline & \multicolumn{2}{|c|}{ Predicted } \\
\hline & & $\mathrm{HC}$ & PD \\
\hline \multirow[t]{2}{*}{ Real } & $\mathrm{HC}$ & 18 & 1 \\
\hline & PD & 0 & 27 \\
\hline \multicolumn{3}{|c|}{ Model accuracy } & $97.8 \%$ \\
\hline \multicolumn{3}{|c|}{ Leave-one-out validation } & $87.0 \%$ \\
\hline \multicolumn{3}{|c|}{ Sensitivity } & $100 \%$ \\
\hline \multicolumn{3}{|c|}{ Specificity } & $94.7 \%$ \\
\hline
\end{tabular}

F

\begin{tabular}{|c|c|c|c|}
\hline & \multicolumn{2}{|c|}{ Predicted } \\
\hline & & PD & MSA \\
\hline \multirow[t]{2}{*}{ Real } & PD & 27 & 0 \\
\hline & MSA & 4 & 4 \\
\hline \multicolumn{3}{|c|}{ Model accuracy } & $88.6 \%$ \\
\hline \multicolumn{3}{|c|}{ Leave-one-out validation } & $76.1 \%$ \\
\hline \multicolumn{3}{|c|}{ Sensitivity } & $50 \%$ \\
\hline \multicolumn{3}{|c|}{ Specificity } & $100 \%$ \\
\hline
\end{tabular}

B

\begin{tabular}{|c|c|c|c|c|c|}
\hline & & \multicolumn{4}{|c|}{ Predicted } \\
\hline & & $\mathrm{HC}$ & PD & MSA & AP-Tau \\
\hline \multirow{4}{*}{ Real } & $\mathrm{HC}$ & 18 & 1 & 0 & 0 \\
\hline & PD & 8 & 27 & 0 & 0 \\
\hline & MSA & 1 & 0 & 7 & 0 \\
\hline & AP-Tau & 2 & 3 & 0 & 4 \\
\hline & & & accur & \multicolumn{2}{|c|}{$88.9 \%$} \\
\hline
\end{tabular}

D

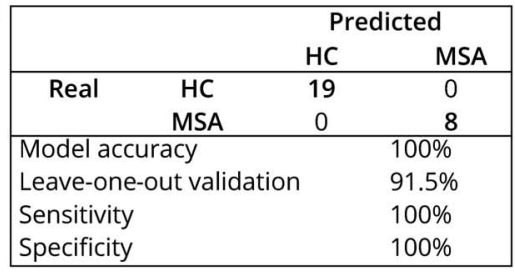

E

\begin{tabular}{|c|c|c|c|}
\hline & \multicolumn{2}{|c|}{ Predicted } \\
\hline & & $\mathrm{HC}$ & AP-Tau \\
\hline \multirow[t]{2}{*}{ Real } & $\mathrm{HC}$ & 19 & 0 \\
\hline & AP-Tau & 4 & 5 \\
\hline \multirow{4}{*}{\multicolumn{3}{|c|}{$\begin{array}{l}\text { Model accuracy } \\
\text { Leave-one-out validation } \\
\text { Sensitivity } \\
\text { Specificity }\end{array}$}} & $85.7 \%$ \\
\hline & & & $82.1 \%$ \\
\hline & & & $55.6 \%$ \\
\hline & & & $100 \%$ \\
\hline
\end{tabular}

I Real diagnosis

\begin{tabular}{|c|c|c|c|}
\hline & & \multicolumn{2}{|c|}{ Predicted } \\
\hline & & PD & AP-Tau \\
\hline \multirow[t]{2}{*}{ Real } & PD & 27 & 0 \\
\hline & AP-Tau & 4 & 5 \\
\hline \multirow{2}{*}{\multicolumn{3}{|c|}{$\begin{array}{l}\text { Model accuracy } \\
\text { Leave-one-out validation }\end{array}$}} & $88.9 \%$ \\
\hline & & & $72.2 \%$ \\
\hline \multicolumn{3}{|c|}{ Sensitivity } & $55.6 \%$ \\
\hline \multicolumn{3}{|c|}{ Specificity } & $100 \%$ \\
\hline
\end{tabular}

\begin{tabular}{|c|c|c|c|}
\hline & & \multicolumn{2}{|c|}{ Predicted } \\
\hline & & MSA & AP-Tau \\
\hline \multirow[t]{2}{*}{ Real } & MSA & 7 & 1 \\
\hline & AP-Tau & 2 & 7 \\
\hline \multicolumn{2}{|c|}{ Model accuracy } & & $82.4 \%$ \\
\hline \multicolumn{3}{|c|}{ Leave-one-out validation } & $72.7 \%$ \\
\hline \multirow{2}{*}{\multicolumn{3}{|c|}{ Sensitivity }} & $77.8 \%$ \\
\hline & & & $87.5 \%$ \\
\hline
\end{tabular}

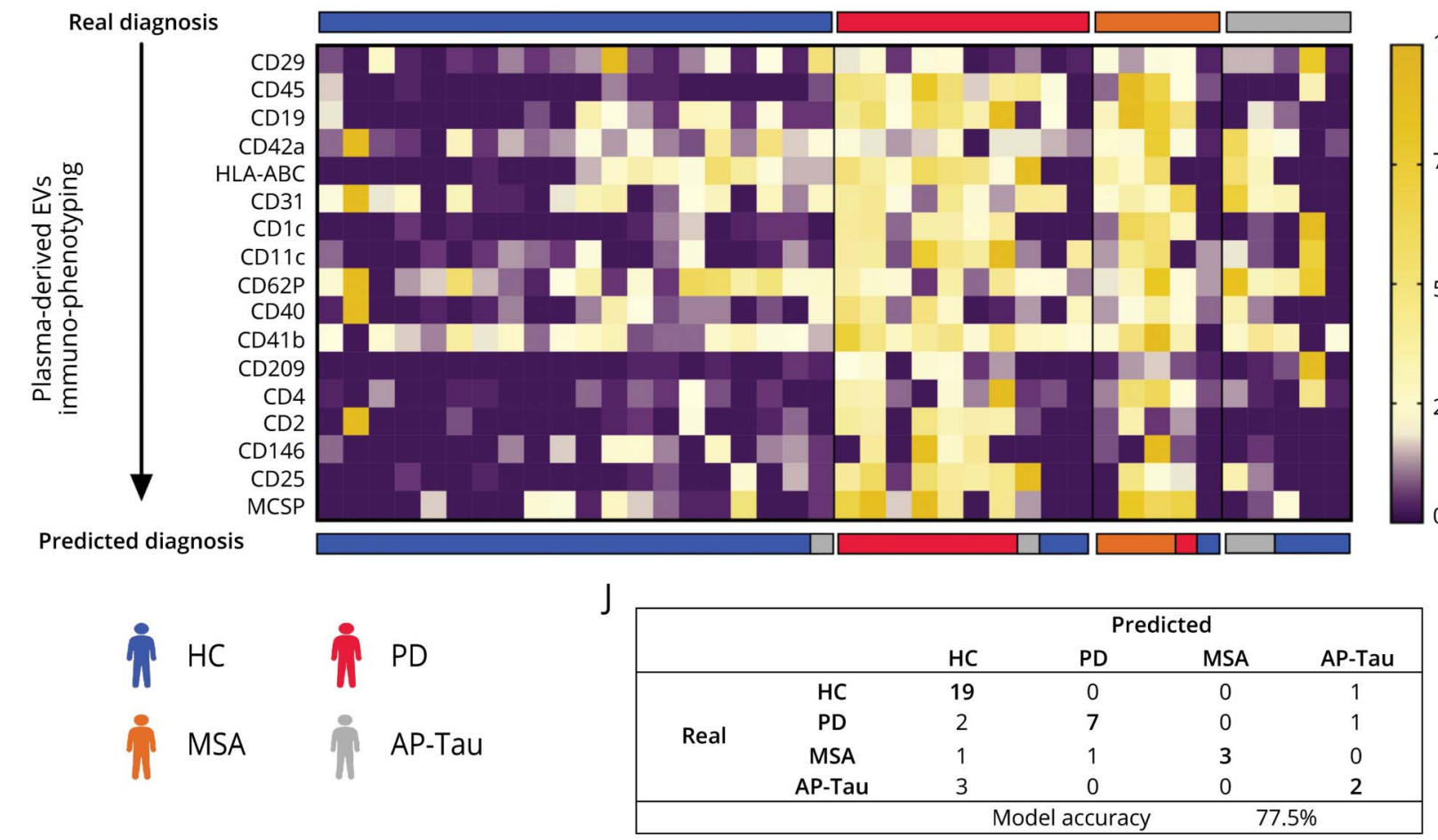

RF modeling to diagnose patients based on the combination of the 17 differentially expressed extracellular vesicle surface markers. (A) Representation of 1 of the 20 different classification trees created by the algorithm to predict the diagnosis PD vs MSA vs AP-Tau vs HC (B-H). Confusion matrix reporting real and predicted diagnosis, accuracy, sensitivity, specificity, and internal validation by the leave-one-out algorithm for each comparison (see Methods). (I) External validation of the RF model; 40 patients were included in the analysis (20 HC, blue; 10 PD, red; 5 MSA, orange; 5 AP-Tau, gray). AP-Tau = atypical parkinsonism with tauopathies; $\mathrm{HC}=$ healthy control; MSA = multiple system atrophy; PD = Parkinson disease.

from Toll-like receptor $4 .^{37,38}$ Moreover a recent multicenter study has shown higher levels of CSF inflammatory biomarkers in PD with dementia and MSA compared with controls and not in AP-Tau vs controls, plus those markers correlated with motor and cognitive impairment. ${ }^{39}$ Likewise, our analysis showed a moderate correlation between CD25, CD146, and cognitive 
impairment in PD suggesting a link between inflammation and a major cognitive decline: CD25 is a costimulatory molecule supporting immune cell activation, ${ }^{40}$ and CD146 acts as an essential regulator of pericyte-endothelial cell communication in the blood-brain barrier and it has been identified as a potential key therapeutic target for cerebrovascular disorders. ${ }^{41}$ In MSA, the concentration of EVs measured by NTA and flow cytometry analysis correlated with disease duration and cognitive impairment. These findings favor the hypothesis of a perpetuation of toxic effects by circulating EVs due to chronic immune activation, even if a compensatory/neuroprotective role of EVs in response to the progressive neurodegeneration cannot be excluded.

Among EV markers differentially expressed in PD, CD146 and MCSP are of interest because they have been associated with melanoma and used for detection of circulating tumor cells. ${ }^{42}$ Consistently, a link between PD and melanoma has been supported by many epidemiologic studies, showing that patients with $\mathrm{PD}$ have a higher incidence of this tumor, even if the underlying pathogenic mechanisms are unknown. ${ }^{43}$

The network analysis of potential interactors of EV surface markers demonstrated that functional pathways and network hubs in PD and MSA were coincident and different from those of AP-Tau. Of interest, among hubs shared by PD and MSA, we found SP1, a transcription factor playing a key role in regulating neuroinflammation in $\mathrm{MS}^{44}$ The most represented KEGG pathways were immune system, signal transduction, signaling molecules and folding, sorting and degradation in alpha-synucleinopathies, whereas FoxO signaling pathway and some pathways of the endocrine system were higher in AP-Tau, matching with the relation that has been found by many authors between endocrine signaling, tauopathies, and FoxO. ${ }^{45,46}$ However, this exploratory network analysis should be interpreted with caution because AP-Tau had less differentially expressed EV markers, consequently the smaller network was a limiting factor to recover potential pathways and functions in tauopathies. Anyhow, among the identified hubs, it has been encouraging to find some of them described in the literature: cytoplasmic protein NCK2 was recently described as a PD-associated gene. ${ }^{47}$ Tyrosine-protein kinase Lyn (LYN), a specific hub of MSA, was related to enhanced microglial migration by aSyn. $^{48}$ Of note, signal transducer and activator of transcription 3 (STAT3), a specific hub of AP-Tau, has been found to be a direct target of C3 and C3a receptor signaling that functionally mediates Tau pathogenesis. ${ }^{49}$ However, these network analyses are hypothetical, and further validation studies are required to assess their possible roles in causing PD and AP.

Limitations of this study are the relatively low number of subjects, especially in AP groups, and the inclusion of patients only with long duration of disease: larger studies and inclusion of different cohorts of patients, especially at early stages of disease, are strongly recommended. Moreover, a customized panel of EV surface proteins including CNS and microglia markers would probably increase the diagnostic model. Finally, this is an antemortem study, and it lacks the diagnostic confirmation of postmortem brain histopathologic analysis.

In conclusion, we systematically characterized circulating EVs in plasma of patients with PD or AP. Several EV surface antigens were differentially expressed and correlated with disease gravity and cognitive impairment, suggesting EVs as potential biomarkers of disease, also in clinical trials for disease-modifying drugs. We propose a diagnostic model built through supervised machine learning algorithms, based on EV-specific signature, which was able to discriminate patients with PD and MSA with high accuracy. Finally, we provided internal and external validations of our model, confirming reliable diagnostic performance.

This is a highly relevant result with a potential impact on clinical practice, allowing with a noninvasive, low-cost blood test to identify patients with PD and MSA. Furthermore, circulating EV surface protein analysis can shed light on the differential inflammation/immunity pathways involved in protein aggregation-related neurodegenerative disease, to be confirmed by functional analysis in experimental models of diseases.

\section{Acknowledgments}

The authors are very grateful to all the patients and their relatives who participated in this study. They are grateful to Mrs. Nicole Vago, research nurse, for her valuable work on the clinical database. They thank the Scientific Research Advisory Board of the Ente Ospedaliero Cantonale (ABREOC) for the financial support of this study.

\section{Study funding}

This study was funded by the Scientific Research Advisory Board of the Ente Ospedaliero Cantonale (ABREOC).

\section{Disclosure}

The authors report no disclosures relevant to the manuscript. Go to Neurology.org/NN for full disclosures.

\section{Publication history}

Received by Neurology: Neuroimmunology \& Neuroinflammation December 16, 2019. Accepted in final form May 28, 2020.

\begin{tabular}{lll} 
Appendix & Authors & \\
\hline Name & Location & Contribution \\
\hline $\begin{array}{l}\text { Elena } \\
\text { Vacchi, MS }\end{array}$ & $\begin{array}{l}\text { Neurocenter of } \\
\text { Southern } \\
\text { Switzerland, Lugano }\end{array}$ & $\begin{array}{l}\text { Acquisition, analysis, and } \\
\text { interpretation of data and drafted } \\
\text { the manuscript }\end{array}$ \\
$\begin{array}{l}\text { Jacopo } \\
\text { Burrello, } \\
\text { MD }\end{array}$ & $\begin{array}{l}\text { Cardiocentro Ticino } \\
\text { Foundation, Lugano }\end{array}$ & $\begin{array}{l}\text { Acquisition, analysis, and } \\
\text { interpretation of data and drafted } \\
\text { the manuscript }\end{array}$ \\
\hline
\end{tabular}


Appendix (continued)

\begin{tabular}{|c|c|c|}
\hline Name & Location & Contribution \\
\hline $\begin{array}{l}\text { Dario di } \\
\text { Silvestre, } \\
\text { MS }\end{array}$ & ITB-CNR, Milan & $\begin{array}{l}\text { Analysis and interpretation of } \\
\text { data }\end{array}$ \\
\hline $\begin{array}{l}\text { Alessio } \\
\text { Burrello, MS }\end{array}$ & $\begin{array}{l}\text { University of } \\
\text { Bologna }\end{array}$ & $\begin{array}{l}\text { Analysis and interpretation of } \\
\text { data }\end{array}$ \\
\hline $\begin{array}{l}\text { Sara Bolis, } \\
\text { MS }\end{array}$ & $\begin{array}{l}\text { Cardiocentro Ticino } \\
\text { Foundation, Lugano }\end{array}$ & $\begin{array}{l}\text { Acquisition and interpretation of } \\
\text { data }\end{array}$ \\
\hline $\begin{array}{l}\text { Pierluigi } \\
\text { Mauri, PhD }\end{array}$ & ITB-CNR, Milan & $\begin{array}{l}\text { Interpreted the data and revised } \\
\text { the manuscript for intellectual } \\
\text { content }\end{array}$ \\
\hline $\begin{array}{l}\text { Giuseppe } \\
\text { Vassalli, MD }\end{array}$ & $\begin{array}{l}\text { Cardiocentro Ticino } \\
\text { Foundation, Lugano }\end{array}$ & $\begin{array}{l}\text { Interpreted the data and revised } \\
\text { the manuscript for intellectual } \\
\text { content }\end{array}$ \\
\hline $\begin{array}{l}\text { Carlo W. } \\
\text { Cereda, MD }\end{array}$ & $\begin{array}{l}\text { Neurocenter of } \\
\text { Southern } \\
\text { Switzerland, Lugano }\end{array}$ & $\begin{array}{l}\text { Interpreted the data and revised } \\
\text { the manuscript for intellectual } \\
\text { content }\end{array}$ \\
\hline $\begin{array}{l}\text { Cinthia } \\
\text { Farina, PhD }\end{array}$ & $\begin{array}{l}\text { San Raffaele } \\
\text { Scientific Institute, } \\
\text { Milan }\end{array}$ & $\begin{array}{l}\text { Interpreted the data and revised } \\
\text { the manuscript for intellectual } \\
\text { content }\end{array}$ \\
\hline $\begin{array}{l}\text { Lucio Barile, } \\
\text { PhD }\end{array}$ & $\begin{array}{l}\text { Cardiocentro Ticino } \\
\text { Foundation, Lugano }\end{array}$ & $\begin{array}{l}\text { Study design, interpreted } \\
\text { the data, and revised the } \\
\text { manuscript for intellectual } \\
\text { content }\end{array}$ \\
\hline $\begin{array}{l}\text { Alain } \\
\text { Kaelin- } \\
\text { Lang, MD, } \\
\text { PhD }\end{array}$ & $\begin{array}{l}\text { Neurocenter of } \\
\text { Southern } \\
\text { Switzerland, Lugano }\end{array}$ & $\begin{array}{l}\text { Study design, patient } \\
\text { enrollment, interpreted the } \\
\text { data, and revised the } \\
\text { manuscript for intellectual } \\
\text { content }\end{array}$ \\
\hline $\begin{array}{l}\text { Giorgia } \\
\text { Melli, MD, } \\
\text { PhD }\end{array}$ & $\begin{array}{l}\text { Neurocenter of } \\
\text { Southern } \\
\text { Switzerland, Lugano }\end{array}$ & $\begin{array}{l}\text { Study design, patient } \\
\text { enrollment, interpreted the } \\
\text { data, and manuscript writing } \\
\text { and revision }\end{array}$ \\
\hline
\end{tabular}

\section{References}

1. Hughes AJ, Daniel SE, Kilford L, Lees AJ. Accuracy of clinical diagnosis of idiopathic Parkinson's disease: a clinico-pathological study of 100 cases. J Neurol Neurosurg Psychiatry 1992;55:181-184.

2. Tolosa E, Wenning G, Poewe W. The diagnosis of Parkinson's disease. Lancet Neurol 2006;5:75-86

3. Thery C, Witwer KW, Aikawa E, et al. Minimal information for studies of extracellular vesicles 2018 (MISEV2018): a position statement of the International Society for Extracellular Vesicles and update of the MISEV2014 guidelines. J Extracell Vesicles 2018;7:1535750.

4. Faure J, Lachenal G, Court M, et al. Exosomes are released by cultured cortical neurones. Mol Cell Neurosci 2006;31:642-648.

5. Goetzl EJ, Boxer A, Schwartz JB, et al. Altered lysosomal proteins in neural-derived plasma exosomes in preclinical Alzheimer disease. Neurology 2015;85:40-47.

6. Thery C, Zitvogel L, Amigorena S. Exosomes: composition, biogenesis and function. Nat Rev Immunol 2002;2:569-579.

7. Sulzer D, Alcalay RN, Garretti F, et al. T cells from patients with Parkinson's disease recognize alpha-synuclein peptides. Nature 2017;546:656-661.

8. Tansey MG, Romero-Ramos M. Immune system responses in Parkinson's disease: early and dynamic. Eur J Neurosci 2019;49:364-383.

9. Koliha N, Heider U, Ozimkowski T, Wiemann M, Bosio A, Wild S. Melanoma affects the composition of blood cell-derived extracellular vesicles. Front Immunol 2016;7: 282.

10. Koliha N, Wiencek Y, Heider U, et al. A novel multiplex bead-based platform highlights the diversity of extracellular vesicles. J Extracell Vesicles 2016;5:29975.

11. Gilman S, Wenning GK, Low PA, et al. Second consensus statement on the diagnosis of multiple system atrophy. Neurology 2008;71:670-676.

12. Hoglinger GU, Respondek G, Stamelou M, et al. Clinical diagnosis of progressive supranuclear palsy: the Movement Disorder Society criteria. Mov Disord 2017;32: 853-864.

13. Armstrong MJ, Litvan I, Lang AE, et al. Criteria for the diagnosis of corticobasal degeneration. Neurology 2013;80:496-503.
14. Kipfer S, Stephan MA, Schupbach WM, Ballinari P, Kaelin-Lang A. Resting tremor in Parkinson disease: a negative predictor of levodopa-induced dyskinesia. Arch Neurol 2011;68:1037-1039.

15. Thery C, Amigorena S, Raposo G, Clayton A. Isolation and characterization of exosomes from cell culture supernatants and biological fluids. Curr Protoc Cell Biol 2006; Chapter 3:Unit 3.22.

16. Dragovic RA, Gardiner C, Brooks AS, et al. Sizing and phenotyping of cellular vesicles using Nanoparticle Tracking Analysis. Nanomedicine 2011;7:780-788.

17. Scardoni G, Tosadori G, Pratap S, Spoto F, Laudanna C. Finding the shortest path with PesCa: a tool for network reconstruction. F1000Res 2015;4:484.

18. Scardoni G, Tosadori G, Faizan M, Spoto F, Fabbri F, Laudanna C. Biological network analysis with CentiScaPe: centralities and experimental dataset integration. F1000Res 2014;3:139.

19. Di Silvestre D, Brambilla F, Scardoni G, et al. Proteomics-based network analysis characterizes biological processes and pathways activated by preconditioned mesenchymal stem cells in cardiac repair mechanisms. Biochim Biophys Acta Gen Subj 2017;1861:1190-1199.

20. Sereni L, Castiello MC, Di Silvestre D, et al. Lentiviral gene therapy corrects platelet phenotype and function in patients with Wiskott-Aldrich syndrome. J Allergy Clin Immunol 2019;144:825-838

21. Huang DW, Sherman BT, Lempicki RA. Systematic and integrative analysis of large gene lists using DAVID bioinformatics resources. Nat Protoc 2009;4:44-57.

22. Burrello J, Burrello A, Stowasser M, et al. The primary aldosteronism surgical outcome score for the prediction of clinical outcomes after adrenalectomy for unilateral primary aldosteronism. Ann Surg Epub 2019 Jan 18.

23. Yang Y, Burrello J, Burrello A, et al. Classification of microadenomas in patients with primary aldosteronism by steroid profiling. J Steroid Biochem Mol Biol 2019;189: 274-282.

24. Vella D, Zoppis I, Mauri G, Mauri P, Di Silvestre D. From protein-protein interactions to protein co-expression networks: a new perspective to evaluate large-scale proteomic data. EURASIP J Bioinform Syst Biol 2017;2017:6.

25. Ohmichi T, Mitsuhashi M, Tatebe H, Kasai T, El-Agnaf OMA, Tokuda T. Quantification of brain-derived extracellular vesicles in plasma as a biomarker to diagnose Parkinson's and related diseases. Parkinsonism Relat Disord 2019;61:82-87.

26. Zhao ZH, Chen ZT, Zhou RL, Zhang X, Ye QY, Wang YZ. Increased DJ-1 and alphasynuclein in plasma neural-derived exosomes as potential markers for Parkinson's disease. Front Aging Neurosci 2018;10:438.

27. Jiang C, Hopfner F, Katsikoudi A, et al. Serum neuronal exosomes predict and differentiate Parkinson's disease from atypical parkinsonism. J Neurol Neurosurg Psychiatry 2020;91:720-729.

28. Tomlinson PR, Zheng Y, Fischer R, et al. Identification of distinct circulating exosomes in Parkinson's disease. Ann Clin Transl Neurol 2015;2:353-361.

29. Tofaris GK. Lysosome-dependent pathways as a unifying theme in Parkinson's disease. Mov Disord 2012;27:1364-1369.

30. Tofaris GK. A critical assessment of exosomes in the pathogenesis and stratification of Parkinson's disease. J Parkinson Dis 2017;7:569-576.

31. Shah R, Patel T, Freedman JE. Circulating extracellular vesicles in human disease. N Engl J Med 2018;379:958-966.

32. Kovacs GG. Molecular pathological classification of neurodegenerative diseases: turning towards precision medicine. Int J Mol Sci 2016;17:189.

33. Gerhard A, Banati RB, Goerres GB, et al. [11C](R)-PK11195 PET imaging of microglial activation in multiple system atrophy. Neurology 2003;61: 686-689.

34. Gerhard A, Pavese N, Hotton G, et al. In vivo imaging of microglial activation with [11C](R)-PK11195 PET in idiopathic Parkinson's disease. Neurobiol Dis 2006;21 404-412.

35. Gerhard A, Trender-Gerhard I, Turkheimer F, Quinn NP, Bhatia KP, Brooks DJ. In vivo imaging of microglial activation with [11C](R)-PK11195 PET in progressive supranuclear palsy. Mov Disord 2006;21:89-93.

36. Gerhard A, Watts J, Trender-Gerhard I, et al. In vivo imaging of microglial activation with $[11 \mathrm{C}](\mathrm{R})-\mathrm{PK} 11195$ PET in corticobasal degeneration. Mov Disord 2004;19: 1221-1226.

37. Balducci C, Frasca A, Zotti M, et al. Toll-like receptor 4-dependent glial cell activation mediates the impairment in memory establishment induced by beta-amyloid oligomers in an acute mouse model of Alzheimer's disease. Brain Behav Immun 2017;60 188-197.

38. La Vitola P, Balducci C, Cerovic M, et al. Alpha-synuclein oligomers impair memory through glial cell activation and via Toll-like receptor 2. Brain Behav Immun 2018;69: 591-602.

39. Hall S, Janelidze S, Surova Y, Widner H, Zetterberg H, Hansson O. Cerebrospinal fluid concentrations of inflammatory markers in Parkinson's disease and atypical parkinsonian disorders. Sci Rep 2018;8:13276.

40. Elgueta R, Benson MJ, de Vries VC, Wasiuk A, Guo Y, Noelle RJ. Molecular mechanism and function of CD40/CD40L engagement in the immune system. Immunol Rev 2009;229:152-172.

41. Chen J, Luo Y, Hui H, et al. CD146 coordinates brain endothelial cell-pericyte communication for blood-brain barrier development. Proc Natl Acad Sci USA 2017; 114:E7622-E7631

42. Rapanotti MC, Campione E, Spallone G, Orlandi A, Bernardini S, Bianchi L. Minimal residual disease in melanoma: circulating melanoma cells and predictive role of MCAM/MUC18/MelCAM/CD146. Cell Death Discov 2017;3:177005.

43. Bose A, Petsko GA, Eliezer D. Parkinson's disease and melanoma: co-occurrence and mechanisms. J Parkinsons Dis 2018;8:385-398. 
44. Menon R, Di Dario M, Cordiglieri C, et al. Gender-based blood transcriptomes and interactomes in multiple sclerosis: involvement of SP1 dependent gene transcription. J Autoimmun 2012;38:J144-J155.

45. Gratuze M, Joly-Amado A, Vieau D, Buee L, Blum D. Mutual relationship between Tau and central insulin signalling: consequences for $\mathrm{AD}$ and tauopathies? Neuroendocrinology 2018;107:181-195.

46. Neri C. Role and therapeutic potential of the pro-longevity factor FOXO and its regulators in neurodegenerative disease. Front Pharmacol 2012;3:15.
47. Sun Y, Ye L, Zheng Y, Yang Z. Identification of crucial genes associated with Parkinson's disease using microarray data. Mol Med Rep 2018;17:3775-3782.

48. Wang $\mathrm{S}, \mathrm{Chu} \mathrm{CH}$, Stewart $\mathrm{T}$, et al. alpha-Synuclein, a chemoattractant, directs microglial migration via H2O2-dependent Lyn phosphorylation. Proc Natl Acad Sci USA 2015;112:E1926-E1935.

49. Litvinchuk A, Wan YW, Swartzlander DB, et al. Complement C3aR inactivation attenuates Tau pathology and reverses an immune network deregulated in tauopathy models and Alzheimer's disease. Neuron 2018;100:1337-1353.e5. 


\section{Neurology \\ Neuroimmunology \& Neuroinflammation}

Immune profiling of plasma-derived extracellular vesicles identifies Parkinson disease

Elena Vacchi, Jacopo Burrello, Dario Di Silvestre, et al.

Neurol Neuroimmunol Neuroinflamm 2020;7;

DOI 10.1212/NXI.0000000000000866

This information is current as of August 12, 2020

\section{Updated Information \& Services}

References

Subspecialty Collections

Permissions \& Licensing

Reprints including high resolution figures, can be found at:

http://nn.neurology.org/content/7/6/e866.full.html

This article cites 47 articles, 4 of which you can access for free at: http://nn.neurology.org/content/7/6/e866.full.html\#\#ref-list-1

This article, along with others on similar topics, appears in the following collection(s):

Parkinson's disease/Parkinsonism

http://nn.neurology.org//cgi/collection/parkinsons_disease_parkinsonis $\mathrm{m}$

Information about reproducing this article in parts (figures,tables) or in its entirety can be found online at:

http://nn.neurology.org/misc/about.xhtml\#permissions

Information about ordering reprints can be found online: http://nn.neurology.org/misc/addir.xhtml\#reprintsus

Neurol Neuroimmunol Neuroinflamm is an official journal of the American Academy of Neurology.

Published since April 2014, it is an open-access, online-only, continuous publication journal. Copyright

Copyright $\odot 2020$ The Author(s). Published by Wolters Kluwer Health, Inc. on behalf of the American Academy of Neurology.. All rights reserved. Online ISSN: 2332-7812.

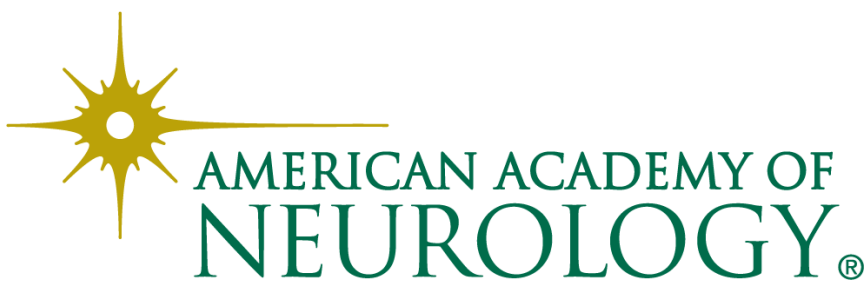

